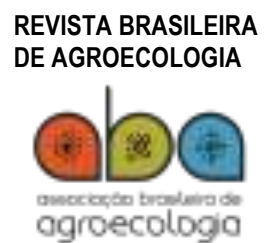

ISSN: $1980-9735$

DOI: $10.33240 /$ rba.v15i5.22871

Vol. 15 | N0 5 | p. $280-290 \mid 2020$

ARTIGO

\title{
IMPACTOS AMBIENTAIS DA IMPLANTAÇÃO DO SISTEMA DE PRODUÇÃO AGROECOLÓGICA INTEGRADA E SUSTENTÁVEL (PAIS) EM UNIDADES FAMILIARES DO DISTRITO FEDERAL
}

\author{
Environmental impacts of the implementation of the Agroecological Integrated and \\ Sustainable Production (PAIS) system in family units of the Federal District
}

Marcela Guzman Munoz ${ }^{1}$, João Paulo Guimarães Soares ${ }^{2}$, Ana Maria Resende Junqueira ${ }^{3}$, Pedro Canuto Macedo Sales ${ }^{4}$

\footnotetext{
${ }^{1}$ Mestre em Agronegócio pela Universidade de Brasília no programa de pós graduação em agronegócioPROPAGA-UNB, e-mail: march_557@hotmail.com

2 Professor do programa de pós graduação em agronegócio PROPAGAUNB e pesquisador da EMBRAPA CERRADOS, e-mail:

jp.soares@embrapa.com

${ }_{3}^{3}$ Coordenadora e Professora do programa de pós graduação em agronegócio PROPAGA-UNB, e-mail: anajunqueiraunb@gmail.com

${ }_{4}$ Mestre em Agronegócio pela Universidade de Brasília no programa de pós graduação em agronegócioPROPAGA-UNB, e-mail: pcsalesadm@gmail.com
}

Recebido em: 27/11/2018 Aceito para publicação em: 22/10/2020

Correspondência para: march_557@hotmail.com

\section{RESUMO}

O objetivo deste estudo foi avaliar os impactos ambientais da implantação da tecnologia Produção Agroecológica Integrada e Sustentável (PAIS) em unidades familiares no Distrito Federal. O estudo foi conduzido em 26 unidades, divididas em três grupos: Grupo A - em conformidade para produção orgânica; Grupo B - em processo de transição agroecológica; Grupo C - de subsistência. Para a coleta de dados utilizou-se o Sistema Produção Sustentável - AMBITEC AGRO, com dados de aspectos gerais, indicadores e variáveis da dimensão ambiental, que atribuíram a cada variável um valor que representou a alteração proporcionada pela implantação da tecnologia. A análise estatística incluiu o cálculo da Percentagem de Impacto Ambiental da Tecnologia (PIT), que reporta, de forma prática, o ganho percentual da tecnologia para cada unidade, e o teste de Wilconxon, identificando os indicadores mais significativos. Foi observado um progresso ambiental com a implantação da tecnologia, obtendo um PIT para o Grupo A de 24,8\%, Grupo B de 21,3\% e Grupo C de 17,3\%, que contribuiu no aumento na qualidade do solo e redução do uso de insumos, impactando positivamente os três grupos avaliados.

Palavras-chave: AMBITEC. Produção Orgânica. Qualidade do solo. Tecnologia PAIS.

\section{ABSTRACT}

The objective of this study was to evaluate the environmental impacts resulted by the implementation of the technology Integrated and Sustainable Agroecological Production (PAIS) in familiar units in Federal District. The study was conducted in 26 production units that were divided into three groups: Group A, in accordance with organic production; Group B, in agroecological transition without certification; and Group C, of subsistence. For data collection, the AMBITEC AGRO - Sustainable Production System was organized in general aspects, indicators and variables of the environmental dimension, which attributed to each variable a value that represented the change provided by the implementation of the technology. The statistical analysis included the calculation of the Percentage of Environmental Impact of Technology (PIT), which reports, in a practical way, the percentage gain of technology of each unit, and the Wilconxon test, identifying the most significant indicators. The environmental evolution was observed with the implementation of the technology, obtaining a PIT for Group A of $24.8 \%$, Group B of $21.3 \%$ and Group C of $17.3 \%$, which contributed to the increase in soil quality and reduction in the use of inputs, positively affecting the three groups evaluated. 
Introdução

A expansão das práticas de produção convencional, baseadas na utilização de padrões tecnológicos de alto consumo de insumos químicos, nos sistemas de produção de alta eficiência e no manejo em monoculturas tem gerado profunda crise no paradigma produtivista (EHLERS, 1999). As dificuldades inerentes a esse enfoque são evidentes, pois, dada a heterogeneidade dos ecossistemas naturais e dos sistemas agrícolas, têm sido criados pacotes tecnológicos homogêneos, pouco respeitosos com o meio ambiente (AQUINO e ASSIS, 2005).

Como resposta a essa situação, no decorrer do tempo surgiram práticas de produção alternativas, que tem como base a Agroecologia (EHLERS, 1999). Agroecologia pode ser entendida como uma ciência ou disciplina que proporciona os fundamentos científicos para apoiar o processo de transição a estilos de agricultura sustentável, incorporando o conhecimento tradicional e aspectos culturais próprios de cada região (ALTIERI et al., 2000).

No entanto, existe, no meio científico, certa confusão entre Agroecologia e sistemas orgânicos de produção (FIGUEIREDO e SOARES, 2012). A Agroecologia é uma ciência que surge ainda na década 1970, como diferentes correntes de agricultura alternativa, de base ecológica, como a biodinâmica, natural, regenerativo, biológico, permacultura e orgânica. Deve-se destacar que, de acordo com a Lei № 10831, de 23 de dezembro de 2003, o conceito de sistema orgânico abrange todas as correntes de agricultura alternativas supracitadas (BRASIL, 2003).

O sistema orgânico se enquadra nos conceitos da Agroecologia, sendo definido como aquele que não permite o uso de "agrotóxicos", medicamentos químicos, hormônios sintéticos e de produtos transgênicos; restringe a utilização de adubos químicos, inclui ações de conservação dos recursos naturais, considera aspectos éticos nas relações sociais internas da propriedade e no trato com os animais (KHATOUNIAN, 2001; FIGUEIREDO e SOARES, 2012; SOUZA et al, 2012; CAPORAL et al, 2011). Portanto, nos sistemas orgânicos, todas as práticas e processos previstos pela ciência agroecológica podem e devem ser aplicados (FIGUEIREDO e SOARES, 2012), desde que em conformidade com a legislação da produção orgânica (Brasil, 2011).

Esses conceitos vêm impulsionando o desenvolvimento de tecnologias sociais de produção dirigidas, principalmente, à agricultura familiar. O Brasil não tem sido alheio a esse tipo de tecnologias, a partir da construção de políticas públicas baseadas em propostas e metodologias reaplicáveis, com princípios de sustentabilidade e em interação com as comunidades (SEBRAE, 2006; ARAÚJO e CÂNDIDO, 2015; VENTURA et al, 2012). No ano 2004, com o apoio da Fundação Banco do Brasil (FBB), o Serviço Brasileiro de Apoio às Micro e Pequenas Empresas - SEBRAE e o Ministério da Integração Nacional (MIN), foi adotada uma tecnologia de produção proposta pelo engenheiro agrônomo africano AlyNdiaye, denominada Produção Agroecológica Integrada e Sustentável - PAIS (SEBRAE, 2006); (PAVAN et al., 2013); (FBB, 2009). Os objetivos principais da concepção dessa tecnologia social foram garantir a subsistência e a segurança alimentar dos produtores familiares, que possuíam pequenas unidades rurais e, de forma secundária, gerar renda através da comercialização do excedente de seu cultivo (FBB, 2009; SEBRAE, 2006).

Os princípios da PAIS incluíram a implantação de um conjunto de práticas para o manejo sustentável da produção, sem a utilização de produtos químicos, promovendo o aproveitamento de recursos existentes na propriedade e na conservação do meio ambiente (PAVAN et al., 2013). A tecnologia PAIS trata-se, basicamente, de um galinheiro central, com piquetes para pastejo em rotação das aves, e um sistema de anéis concêntricos de culturas diversificadas, conquanto que o mesmo seja sustentável, que promova a recuperação e conservação do meio ambiente, e que tenha uma permanente retroalimentação (CORDEIRO et al., 2010; BERTONI, 2011). Deve-se destacar que um dos primeiros estados brasileiros que adotou a tecnologia PAIS foi o Distrito Federal, promovendo sua implantação principalmente nos assentamentos e estreitamente com agricultores familiares (SEBRAE, 2006). De acordo com informação do SEBRAE, no DF existem pouco mais de 160 unidades implantadas e em implantação.

Salienta-se que, quando uma tecnologia é introduzida, ampliada ou modificada, gera impactos na complexa natureza das interações socioculturais e ambientais, os quais implicam em incertezas sobre as possíveis repercussões da inovação implantada. A tecnologia social PAIS, ao se sustentar em princípios 
agroecológicos, pressupõe a manutenção e recuperação do meio ambiente, constituindo um aspecto central a ser atendido sob os princípios da sustentabilidade (RODRIGUES, 2006).

Para avaliação dos impactos ambientais (AIA) das atividades relacionadas à agricultura familiar e à sustentabilidade, o método escolhido necessita atender a grande variedade de atividades agrícolas e não agrícolas desenvolvidas nas mais variadas condições. Segundo Rodrigues (2006), o método para uma avaliação adequada deve ser apropriado para guiar a escolha de atividades, tecnologias e formas de manejo de acordo com as potencialidades e restrições de uso do espaço rural e de sua inserção nos objetivos de desenvolvimento local sustentável. Esses sistemas de AIA mais contextualizados com as características do meio a serem aplicados, estão sendo desenvolvidos pela Empresa Brasileira de Pesquisa Agropecuária (EMBRAPA) para balizar a adoção de inovações tecnológicas agropecuárias (Sistema Ambitec-Agro) e para a gestão ambiental de atividades das unidades de produção rural. O sistema constitui uma ferramenta aplicável a processos de certificação ambiental, contribuindo para o desenvolvimento rural sustentável (BARRETO, 2010).

O presente trabalho utilizou o sistema Ambitec-Agro, versão para a avaliação da produção sustentável, que se baseia numa experiência prévia de AIA, aplicada a projetos de pesquisa no âmbito institucional. Nessa experiência foi selecionado e validado um conjunto de indicadores direcionados à avaliação ex-ante e ex-post da contribuição de uma inovação tecnológica para o desempenho ambiental da atividade agropecuária, a partir da adaptação desses indicadores na plataforma MS-Excel (RODRIGUES et al., 2003).

Nesse sentido, o objetivo deste trabalho foi avaliar os impactos ambientais gerados pela implantação da tecnologia social PAIS em unidades familiares do Distrito Federal, com a utilização do sistema Ambitec-Agro, versão para a avaliação da produção sustentável.

\section{Material e Métodos}

\section{Caracterização das propriedades}

O estudo foi conduzido em quatro regiões administrativas do Distrito Federal: Sobradinho, Planaltina, Brazlândia, e São Sebastião. A escolha dessas regiões baseou-se no fato de estarem presentes no assentamento Chapadinha, o mais antigo de Brasília-DF, por serem produtores interessados em avançar na produção orgânica, onde a tecnologia PAIS já havia sido aplicada a algum tempo, de modo a permitir a análise ex-ante e ex-post. O levantamento de dados foi realizado no ano 2014, durante o período compreendido entre os meses de março e agosto, por meio de visitas in loco e entrevistas. Foram escolhidas vinte e quatro unidades de agricultura familiar seguindo, além do critério de localização, o fato de serem unidades que já adotavam a tecnologia PAIS e estavam presentes no levantamento do Sebrae (2006).

Para avaliação, as unidades foram divididas em três grupos de agricultores familiares, classificados de acordo com o modo produtivo utilizado e sua inserção no mercado. $O$ grupo $A$ incluiu produtores PAIS, com declaração do cadastro do produtor orgânico vinculado à OCS, com participação ativa em mercados de venda direta. $O$ grupo $B$ abrangeu produtores PAIS em processo de transição agroecológica e participação no mercado de venda direta. $\mathrm{O}$ grupo $\mathrm{C}$ era formado com produtores PAIS de subsistência, ou seja, que destinavam sua produção principalmente para o autoconsumo, com baixa inserção de seus produtos ao mercado, e que iniciam a implementação de modelos agroecológicos em suas propriedades.

No grupo $A$, as principais atividades dos agricultores familiares fazem parte da região de Sobradinho, antes da implantação da tecnologia social PAIS, baseavam-se na produção de monoculturas de milho e soja, com utilização de insumos químicos. Sua inserção na tecnologia PAIS aconteceu em 2010 - 2011 e, em reflexo disso, já no ano de 2012, dez produtores (incluindo os oito avaliados) receberam avaliação de conformidade como produtores orgânicos via Organismos de Controle Social.

No grupo B, em que os produtores estão localizados nas regiões de Planaltina e São Sebastião, verificou-se que antes da implantação da tecnologia social PAIS, eram produtores de subsistência e cultivavam pequenas áreas para a produção de milho e mandioca, com uso de insumos químicos em 
baixas quantidades. A adoção da tecnologia PAIS, em ambos os grupos A e B, impulsionou a incorporação, principalmente a produção de hortaliças, galinhas e ovos, sob princípios orgânicos.

Os produtores do grupo $\mathrm{C}$, pertencentes à região de Brazlândia, informaram que suas principais atividades, antes de ter inserido a tecnologia PAIS, baseavam-se na produção convencional de morango, milho e feijão, com uso de insumos químicos. A implantação da tecnologia nesse grupo de produtores tem sido a mais recente, abarcando o período 2012 e 2013.

\section{Análise de dados}

O instrumento de coleta de dados para a avaliação dos impactos ambientais utilizado na pesquisa foi o sistema Ambitec Agro - versão para a avaliação da produção sustentável - (SOARES et al., 2015; SOARES e RODRIGUES, 2013).

O Ambitec Agro é um programa formado por um conjunto de planilhas eletrônicas (MS-EXCEL ${ }^{\circledR}$ ) constituídas por indicadores ambientais. A aplicação do instrumento incluiu o desenvolvimento de uma entrevista in loco com o produtor, com a finalidade de avaliar sua percepção a partir de suas vivências e experiências em relação ao impacto ambiental gerado da implantação da tecnologia social PAIS. O processo de coleta de dados foi feito em duas etapas e incluiu o preenchimento de duas planilhas distintas do sistema Ambitec. Primeiramente, o produtor respondeu questões relacionadas com a sua situação anterior à implantação da tecnologia (ex-ante), quando suas atividades de produção eram convencionais; e, na segunda etapa, buscou-se avaliar sua percepção à respeito situação atual, um a quatro anos após implantação da tecnologia PAIS (ex-post). As duas etapas foram realizadas no mesmo dia, fazendo apenas a diferenciação com relação às duas formas de produção.

O sistema Ambitec, na avaliação de impactos ambientais, compõe-se de dois aspectos gerais a serem ponderados: (a) o primeiro se relaciona com o uso de insumos e recursos, e (b) o segundo com o uso de insumos veterinários e matérias primas (IRIAS et al., 2004). Cada um desses aspectos está formado por indicadores, e cada indicador é constituído por uma série de variáveis. Podem-se relacionar de forma consecutiva os aspectos, indicadores e variáveis que constituem a avaliação de impactos ambientais via Ambitec na Figura 1. 


\section{AVALIAÇÃO DE IMPACTO AMBIENTAL}

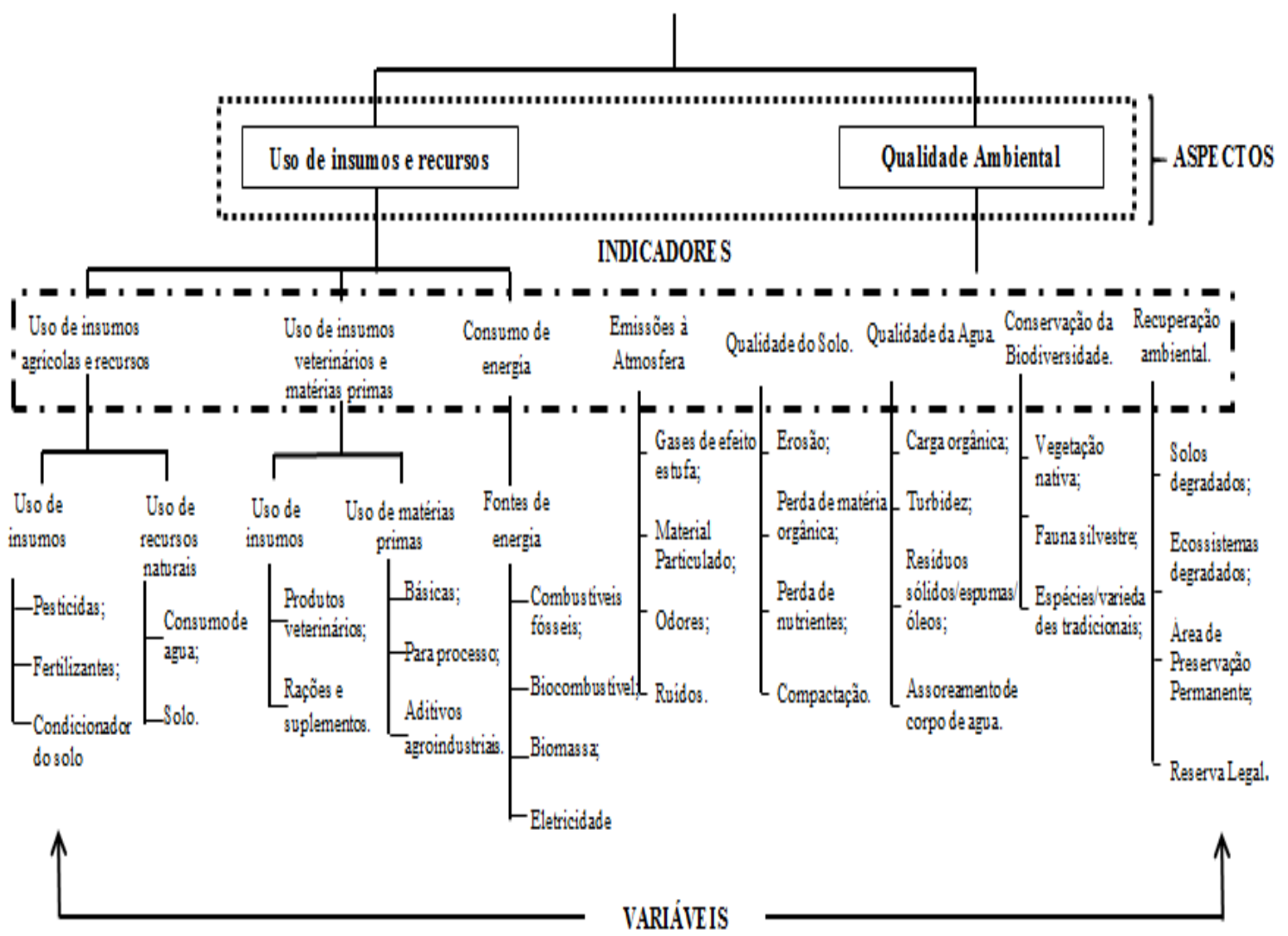

Figura 1. Adaptado de Soares e Rodrigues (2013).

Cada uma das variáveis tem um fator de ponderação $(k)$, que indica o peso ou importância de cada uma delas. O programa inclui, também, a escala geográfica de ocorrência da alteração do componente do indicador, determinando a abrangência do impacto em três grupos: pontual, local e entorno. É considerado pontual, quando o efeito se restringe ao ambiente de implantação da tecnologia; local, quando o efeito se faz sentir fora do ambiente da tecnologia, mas restrito aos limites da unidade produtiva; entorno, quando o impacto gerado ultrapassa os limites da unidade produtiva (RODRIGUES et al., 2003).

As variáveis de cada um dos indicadores são mensuradas com base no coeficiente de alteração do indicador, entendido como o impacto da atividade sob as condições de manejo específicas para cada variável. Os coeficientes de alteração podem variar de $-3 a+3$, sendo que o coeficiente +3 indica uma grande influência positiva no componente; +1 , moderada influência positiva no componente; 0 , componente inalterado; -1 , moderada influência negativa no componente e -3 , grande influência negativa no componente (SOARES e RODRIGUES, 2013). Os coeficientes de alteração foram inseridos segundo a percepção do produtor, que foi quem identificou o grau de impacto para cada variável. Uma vez inserido o coeficiente de alteração, automaticamente o programa gerou o coeficiente de impacto parcial que, sumarizados entre sim, conformam o impacto total do indicador.

$\mathrm{Na}$ matriz de resultados do sistema Ambitec, foram agrupados e consolidados para cada produtor avaliado os indicadores do impacto ambiental, tanto antes da implantação da tecnologia, quanto depois, os quais tiveram uma representação gráfica, gerado um índice de impacto geral com uma variação de +15 a -15, dependendo do direcionamento do impacto, se benéfico ou deletério, respectivamente.

A metodologia de cálculo do percentual de impacto da tecnologia (PIT) foi desenvolvida visando prover uma análise comparativa entre as condições ambientais anteriores e posteriores à adoção tecnológica. Além disso, objetivou-se caracterizar, também de forma prática, o ganho percentual da 
tecnologia para cada unidade produtiva, ou seja, com fácil entendimento ao produtor familiar em evidenciar as diferenças em termos de coeficientes técnicos do processo e as mudanças proporcionadas pela tecnologia adotada (SOARES et al., 2015).

Nesse sentido, calculou-se, inicialmente, o Percentual de Impacto da Tecnologia (PIT) para cada unidade familiar, para, depois, fazer uma média das unidades avaliadas (SOARES et al., 2015; SOARES E RODRIGUES, 2013). A medida pode assumir valores positivos ou negativos, indicando a direção: se o índice de impacto mensurado entre os dois momentos (antes e após a introdução da tecnologia) foi crescente ou decrescente, respectivamente. Segue a descrição do cálculo:

$P I T_{i}=\left(\frac{\mu_{2 i}-\mu_{1 i}}{A M}\right) \times 100$

Sendo:

$P I T_{i} P I T_{i}$ : Percentagem de Impacto da Tecnologia do indivíduo i, i=1

$\mu_{2 i} \mu_{2 i}$ : Índice de impacto (ambiental) depois da introdução da tecnologia, referente ao indivíduo i;

$\mu_{1 i} \mu_{1 i i}$ Índice de impacto (ambiental) antes da introdução da tecnologia, referente ao indivíduo i;

AMAM: Amplitude máxima possível da escala Ambitec (= 30).

Depois de estabelecido o PIT, houve a necessidade de fazer uma discussão sobre a contribuição individual de cada um dos indicadores na geração deste impacto. Para isso, aplicou-se uma análise estatística, com o intuito de estabelecer se existiram diferenças significativas entres os indicadores dos impactos ambientais avaliados. Essa análise foi feita usando o teste não paramétrico de Wilcoxon para amostras emparelhadas, ao nível de significância de 5\%.

\section{Resultados e Discussão}

Os três grupos avaliados apresentaram influência ambiental positiva a partir da implantação da tecnologia PAIS, a qual foi evidenciada a partir do cálculo do Percentual de Impacto da Tecnologia (PIT) que se relaciona na Tabela 1. A tecnologia de Produção Agroecológica Integrada e Sustentável (PAIS) caracteriza-se por ser uma proposta que estimula o desenvolvimento de práticas agrícolas menos impactantes no meio ambiente baseadas no cultivo orgânico, bem como na otimização do uso sustentável dos recursos naturais da propriedade (ROMÃO, 2010).

Tabela 01. Índice médio de impacto ambiental antes e depois do PAIS e percentagem de impacto da tecnologia (PIT) para cada grupo.

\begin{tabular}{ccccc}
\hline GRUPO & $\begin{array}{c}\text { Média impacto } \\
\text { ambiental antes do } \\
\text { PAIS }\end{array}$ & $\begin{array}{c}\text { Média impacto } \\
\text { ambiental depois } \\
\text { do PAIS }\end{array}$ & Diferenciação & PIT Ambiental (\%) \\
\hline A & $-3,87$ & 3,57 & 7,43 & 24,8 \\
B & $-3,40$ & 2,99 & 6,39 & 21,3 \\
C & $-2,22$ & 2,96 & 5,18 & 17,3 \\
\hline
\end{tabular}

Fonte. Dados da pesquisa, coletados entre os meses de março e agosto de 2014.

Considerando o PIT positivo nos três grupos avaliados, foi dado destaque aos indicadores que tiveram maior significância estatística em cada um deles.

O grupo A destacou-se como o mais influenciado positivamente pela implantação da tecnologia PAIS na dimensão ambiental, uma vez que promoveu um incremento de $24,8 \%$ no índice de impacto ambiental médio. $O$ grupo A proporcionou, ainda, redução de alguns dos coeficientes dos indicadores que 
concorriam para a degradação do ambiente com destaque para as variáveis, utilização de insumos agrícolas, medicamentos veterinários e qualidade do solo.

No aspecto uso de insumos agrícolas e recursos, o indicador que apresentou maior variação $(P<0,05)$ foi o de uso de insumos agrícolas e recursos, sendo antes da implantação do sistema PAIS de $\mu=$ $-3,8$, passando para $\mu=9,3$. Este indicador relacionou as seguintes variáveis: uso de insumos como pesticidas, fertilizantes, condicionadores de solo; e as variáveis uso de recursos naturais, como consumo de água e aproveitamento do solo.

O estudo verificou que os produtores do Grupo A, certificados pelos Organismos de Controle Social (OCS), eliminaram completamente o uso de insumos químicos na sua propriedade, e tornaram sua produção orgânica por meio do reaproveitamento de resíduos naturais e estercos, como adubos, além da utilização da associação de cultura e utilização de caldas para o controle de pragas e insetos.

Essa prática é fundamental na conservação do meio ambiente, pois, segundo Costabeber (1998), na agricultura convencional os efeitos dos insumos químicos sobre os recursos naturais são refletidos na degradação dos ecossistemas e recursos produtivos, comprometendo a produtividade no longo prazo, tanto das gerações presentes, quanto das próximas. Este resultado foi similar ao observado no estudo de Rodrigues et al. (2006), no qual foi usada a metodologia Ambitec na avaliação socioambiental da integração tecnológica Embrapa pecuária sudeste para produção leiteira na agricultura familiar. Os autores ressaltam que um dos indicadores que se destacou positivamente foi o uso de insumos agrícolas e recursos, com um coeficiente de impacto médio igual a 10,5.

Quanto às variáveis do uso de recursos naturais como consumo de água e aproveitamento do solo, os produtores ressaltaram que os procedimentos de produção orgânica implementados após a inserção tecnologia PAIS permitiram uma melhor organização e aproveitamento desses recursos. Notouse maior reaproveitamento de resíduos para adubação orgânica, como também técnicas de manejo do solo que diminuem o consumo de água para as plantas.

Ocorreram, também, diferenças estatísticas significativas para o indicador uso de insumos veterinários e matérias-primas (sendo antes da implantação do PAIS igual a $\mu=-3,5$, passando para $\mu=$ 6,8). Essa diminuição pode ser justificada pela mudança ocorrida no tratamento das doenças dos animais, uma vez que, antes, os produtores utilizavam de medicamentos químicos e, após a implantação do PAIS e ingresso na produção orgânica, passou a ser feito o manejo preventivo de forma a se evitar doenças. Foram, ainda, autorizados apenas o uso produtos homeopáticos e fitoterápicos (BRASIL, 2011), enquanto na alimentação se faz uso de alimentos produzidos na propriedade, já que a alimentação fornecida devesse ser orgânica e dificilmente é encontrada no mercado.

Já no aspecto qualidade ambiental, os indicadores que tiveram maior destaque foram conservação da biodiversidade (sendo antes da implantação do PAIS igual a $\mu=-4,6$, passando para $\mu=$ 8,2 ), qualidade do solo (sendo antes da implantação do PAIS igual a $\mu=-6,4$, passando para $\mu=8,8$ ), e recuperação ambiental (sendo antes da implantação do PAIS igual a $\mu=-3,4$, passando para $\mu=4,1$ ). A adoção da tecnologia PAIS significou um aumento na diversidade de culturas e atividades produtivas. Conforme mencionado por Barbosa (2013), o planejamento do sistema produtivo sob o princípio da biodiversidade, com novas práticas de manejo, que priorizam pelo reaproveitamento de insumos naturais do próprio ambiente, com o objetivo de cumprir com a base agroecológica e sustentável da tecnologia, pode ser apontado como principal influenciador das modificações observadas nas propriedades.

O indicador qualidade do solo foi, também, importante pois, segundo a percepção dos produtores avaliados, a tecnologia PAIS propiciou o conhecimento e desenvolvimento de práticas conservacionistas do solo, como a eliminação de adubos químicos e agrotóxicos nas práticas produtivas. Resultado que acompanha ao objetivo da criação da tecnologia, que, segundo Pavan et al. (2013), tem entre os principais objetivos o desenvolvimento produtivo, a partir dos recursos disponíveis na própria propriedade.

Esse resultado demonstra a capacidade da tecnologia PAIS em gerar benefícios para manutenção do solo, uma vez que a utilização de adubos químicos e agrotóxicos destrói a vida útil do solo. Sabe-se que agrotóxicos e adubos químicos de alta solubilidade prejudicam a disponibilidade de nutrientes para as plantas, matam minhocas, besouros e outros pequenos organismos altamente benéficos para a agricultura, além de aumentar o poder de ação e reprodução de insetos que sobrevivem a uma 
pulverização, aumentando sua a resistência genética contra o veneno, conforme Meirelles e Rupp (2005). Destacou-se também a adubação verde, com o emprego de espécies de plantas fixadoras de nitrogênio atmosférico, considerado como um dos pilares da produção orgânica (KHATOUNIAN, 2001), e uma maior rotação de culturas.

O resultado de nosso estudo tem sido observado de modo semelhante por outras pesquisas nas quais o sistema Ambitec foi empregado para avaliação de impactos ambientais. Em Barreto (2010), por exemplo, que estudou o manejo agroecológico da caatinga em unidades de produção familiar no Oeste Potiguar, foi encontrado que a maior contribuição foi proporcionada pela melhoria da capacidade produtiva do solo com diferenciação de $\mu=11,43$. Neves (2012), avaliando as estratégias para produção de carne bovina orgânica no Brasil, verificou que o índice que obteve a maior variação foi o índice qualidade do solo, cuja variação foi de $\mu=15,56$.

Os indicadores "emissões à atmosfera" não apresentaram diferenças estatisticamente significativas na comparação antes - depois da implantação da tecnologia. Realidade que pode ser explicada devido ao fato de os produtores reconhecerem que, antes de implantar a tecnologia PAIS, suas atividades de produção eram muito simples e fundamentalmente manuais. Não faziam uso do trator, nem de grandes quantidades de insumos químicos, não havendo, dessa forma, muitas modificações após a implantação.

No que se refere à qualidade da água, os produtores sinalizaram que não tem evidenciado grandes mudanças com a implantação do sistema PAIS, o que também pode ser justificado pela realidade de uso da água antes de sua implantação.

O Grupo B apresentou uma percentagem de impacto ambiental (PIT) de $21,3 \%$, o que significou uma contribuição positiva de ter implantado a tecnologia PAIS, embora numa menor proporção comparado com o Grupo A. Observou-se convergências com o Grupo A, em relação aos indicadores com maior diferenciação estatística, embora sua significância fora menos expressiva. Esses indicadores foram, no aspecto uso de insumos e recursos: uso de insumos agrícolas e recursos, e uso de insumos veterinários; e, no aspecto qualidade ambiental: qualidade do solo, conservação da biodiversidade e recuperação ambiental.

$O$ indicador mais expressivo foi o relacionado com o uso de insumos agrícolas e recursos, sendo antes da implantação do PAIS igual a $\mu=-3,0$, passando para $\mu=5,9$, pois $88 \%$ dos produtores $(7 / 8)$ diminuíram significativamente 0 uso de insumos químicos e estão trabalhando sob princípios agroecológicos.

Em relação ao consumo de recursos naturais, apesar de três produtores reconhecerem que o consumo da água aumentou com a implantação da tecnologia, isso pode ser explicado pela mudança na realidade produtiva desses estabelecimentos antes e depois do PAIS, uma vez que aumentou as áreas cultivadas como também a variabilidade de produtos. Os cinco produtores restantes, pelo contrário, ressaltaram que a tecnologia diminuiu consideravelmente o consumo da água, porque continuaram a cultivar a mesma área que antes do sistema PAIS, e usavam outros sistemas de irrigação, como aspersores de alta pressão, e o consumo era maior. Com o PAIS, foi implantado o sistema de irrigação por gotejamento, que diminuiu consideravelmente o consumo de água.

$O$ indicador uso de insumos veterinários e matérias-primas, ainda que seja significativo estatisticamente, é menos expressivo, sendo antes da implantação do PAIS igual a $\mu=-3,0$, passando para $\mu=4,2$, pois os produtores reconheceram que, embora tenham diminuído o uso de insumos veterinários em seus animais, ainda empregam alguns produtos para prevenir, controlar ou curar doenças.

Adicionalmente, devido ao aumento no número de aves que inclui a tecnologia, ocorreu um aumento na aquisição de rações e suplementos fora da propriedade, uma vez que não é possível ser autossustentável para todos os alimentos necessários. Porém, destaca-se que os produtos adquiridos fora das propriedades seguem os mesmos padrões daqueles produzidos internamente.

Nos impactos qualidade do solo, conservação da biodiversidade e recuperação ambiental, os produtores relataram o impacto positivo da implantação do sistema PAIS e suas percepções convergiram com as fornecidas pelo Grupo A. Da mesma forma, os indicadores emissões à atmosfera e qualidade da 
água não apresentaram diferenças estatisticamente significativas na comparação antes - depois da implantação da tecnologia.

No Grupo C, ainda que seja o mais novo a implantar a tecnologia, ficou evidente avanço importante no aspecto ambiental, apresentando um PIT de 17,3\%. Houve indicadores que tiveram diferenças significativas, mas foram muito menos expressivos comparados com os Grupos A e B.

Os indicadores uso de insumos agrícolas, sendo antes da implantação do PAIS igual a $\mu=-2,9$, passando para $\mu=2,3$, e recursos e uso de insumos veterinários e matérias-primas (sendo antes da implantação do PAIS igual a $\mu=-3,0$, passando para $\mu=2,1$ ), tiveram diferenciação, pois os produtores, em processo de transição agroecológica, buscam eliminar qualquer insumo químico de sua propriedade, incluídos os produtos veterinários. No entanto, eles relataram ser um processo complexo, pois têm a percepção de que o controle de pragas nas lavouras e doenças nos animais são mais difíceis de manejar de forma orgânica.

Outro indicador importante foi a qualidade do solo, sendo antes da implantação do PAIS igual a $\mu$ $=-3,1$, passando para $\mu=3,5$, pois, com as poucas práticas que os produtores têm empregado, têm sido evidenciadas melhorias significativas. Os indicadores conservação da biodiversidade, recuperação ambiental, emissões à atmosfera e qualidade da água não apresentaram diferença estatística significativa.

Os indicadores ambientais que tiveram maior significância estatística na diferenciação antes depois da implantação da tecnologia, nos três grupos, e os que não tiveram influência na avaliação são apresentados na Tabela 2.

Tabela 02. Indicadores ambientais mais representativos dos grupos A, B e C e sua diferenciação na comparação ex ante - ex post em propriedades do Distrito Federal.

\begin{tabular}{|c|c|c|c|c|c|c|c|c|c|c|}
\hline \multirow[t]{2}{*}{ Aspecto } & \multirow{2}{*}{$\begin{array}{l}\text { Indicador } \\
\text { ambiental }\end{array}$} & \multicolumn{3}{|c|}{$\begin{array}{c}\boldsymbol{\mu} \text { Indicador } \\
\text { Grupo A }\end{array}$} & \multicolumn{3}{|c|}{$\begin{array}{c}\mu \text { Indicador } \\
\text { Grupo B }\end{array}$} & \multicolumn{3}{|c|}{$\begin{array}{c}\mu \text { Indicador } \\
\text { Grupo C }\end{array}$} \\
\hline & & Antes & Depois & \# & Antes & Depois & \# & Antes & Depois & \# \\
\hline \multirow{3}{*}{$\begin{array}{c}\text { Uso de } \\
\text { insumos } \\
\text { e } \\
\text { recursos }\end{array}$} & $\begin{array}{l}\text { Uso de } \\
\text { insumos } \\
\text { agrícolas e } \\
\text { recursos }\end{array}$ & $-3,8$ & 9,3 & $13,1^{*}$ & $-3,0$ & 5,9 & $8,9 *$ & $-2,9$ & 2,3 & $5,2 *$ \\
\hline & $\begin{array}{l}\text { Uso de } \\
\text { insumos } \\
\text { veterinários }\end{array}$ & $-3,5$ & 6,8 & $10,3 *$ & $-3,0$ & 4,2 & $7,2 *$ & $-3,0$ & 2,1 & $5,1^{*}$ \\
\hline & $\begin{array}{l}\text { Consumo } \\
\text { de energia }\end{array}$ & & & & & NS & & & & \\
\hline \multirow{5}{*}{$\begin{array}{l}\text { Qualida } \\
\text { de } \\
\text { ambient } \\
\text { al }\end{array}$} & $\begin{array}{l}\text { Emissões à } \\
\text { atmosfera }\end{array}$ & & & & & NS & & & & \\
\hline & $\begin{array}{l}\text { Qualidade } \\
\text { do solo }\end{array}$ & $-6,4$ & 8,8 & $15,2 *$ & $-4,3$ & 6,9 & $11,2 *$ & $-3,1$ & 3,5 & $6,6^{*}$ \\
\hline & $\begin{array}{l}\text { Qualidade } \\
\text { da água }\end{array}$ & & & & & NS & & & & \\
\hline & $\begin{array}{l}\text { Conservaçã } \\
\text { o } \\
\text { biodiversid } \\
\text { ade }\end{array}$ & $-4,6$ & 8,2 & $12,8^{*}$ & $-4,2$ & 5,4 & $9,6 *$ & & NS & \\
\hline & $\begin{array}{l}\text { Recuperaçã } \\
\text { o ambiental }\end{array}$ & $-3,4$ & 3,1 & $6,5^{*}$ & $-1,5$ & 1,9 & $3,4 *$ & & NS & \\
\hline
\end{tabular}

Fonte: Dados da pesquisa, coletados entre os meses de março e agosto de 2014

No que se refere ao impacto ambiental, foram evidenciados indicadores comuns de melhoria dos índices para os três grupos influenciados positivamente pela implantação da tecnologia PAIS. Houver uma redução importante no uso de insumos agrícolas, recursos e produtos veterinários, além de um aumento na qualidade do solo ao se avaliar as condições existentes antes e depois da implantação da tecnologia PAIS. 
Foram identificados três indicadores que, segundo a percepção dos produtores avaliados, não foram influenciados pela implantação do sistema PAIS, consumo de energia, emissões à atmosfera e a qualidade da água. Estes podem ser considerados aspectos mais difíceis de mensurar, necessitando o desenvolvimento de testes específicos, uma vez que a avaliação através do Ambitec Agro ocorre apenas através da percepção dos produtores.

\section{Conclusões}

É possível com auxílio do Ambitec Agro, verificar que os produtores estão adotando práticas produtivas conservacionistas, por meio da implantação da tecnologia social PAIS, o que tem gerado impactos positivos nas variáveis ambientais. Deve-se destacar a importância de se trabalhar segundo os princípios da Agroecologia, e a participação desses agricultores em organismos de controle para a avaliação da conformidade orgânica, o que faz com que os produtores vinculados à OCS (Grupo $A$ ) tenham maior percentagem de impacto ambiental. A partir da implantação da tecnologia PAIS, têm sido adotadas práticas como a elaboração de compostagem, adubação verde e rotação de culturas, favorecendo a conservação e enriquecimento do solo. Foram observadas melhorias ambientais no que se refere à qualidade do solo, consumo de água, redução do uso de medicamentos e insumos químicos. Realidade, essa, que culminou no desenvolvimento de ambientes produtivos mais equilibrados e biodiversos nas propriedades pesquisadas. De forma geral, a tecnologia social PAIS constitui-se como uma alternativa de produção viável para os agricultores familiares.

\section{Agradecimentos}

Aos produtores rurais, pela valiosa contribuição à pesquisa. Às instituições do DF, SEBRAE, EMATER e IBRAM, pelo apoio logístico e à Universidade de Brasília e ao Programa de Pós-Graduação em Agronegócios (PROPAGA), pela oportunidade de realização da pesquisa.

\section{Referências}

ALTIERI, M; NICHOLLS, C. Agroecología: Teoría y práctica para una agricultura sustentable. Programa de las Naciones Unidas para el Medio Ambiente.Red de Formación Ambiental para América Latina y el Caribe.México, 2000.

ARAÚJO, R. O. A; CÂNDIDO, G. A. Tecnologia social e inovação social: interação indutora do desenvolvimento sustentável nos territórios rurais. Revista Espacios, v. 36, n. 132015.

AQUINO, A. M; ASSIS, R. L. Agroecologia: princípios e técnicas para uma agricultura orgânica sustentável. Embrapa Informação tecnológica. Brasília - DF, 2005.

ASSIS, R. L. DE; ROMEIRO, A. R. Agroecologia e agricultura orgânica: controvérsias e tendências. Desenvolvimento e Meio Ambiente, v. 6, n. 6, p. 67-80, 2002.

BARBOSA, L. K. Produção agroecologia integrada e sustentável no município de Mogeiro - PB. Trabalho de conclusão de curso (Geografia). Universidade Estadual de Paraibá, 2013.

BARRETO, H. F. Impacto do manejo agroecológico da caatinga em unidades de produção familiar no oeste potiguar. Dissertação (Mestrado em Ciências Animais) UFERSA,2010.

BERTONI, F. M. A tecnologia social de produção agroecológica integrada e sustentável - PAIS - na comunidade quilombola chácara do Buriti - Campo Grande/MS. Dissertação (Desenvolvimento Local) - Universidade Católica Dom Bosco, 2011.

BRASIL. Lei no 10.831, 23 de dezembro de 2003. Dispõe sobre agricultura orgânica e dá outras providências. Presidência da República Casa Civil Subchefia para Assuntos Jurídicos, p. 8, 2003.

BRASIL. Instrução normativa no 46, de 06 de outubro de 2011. Diário Oficial da República Federativa do Brasil, Poder Executivo, Brasília, DF, 06 outubro. 2011. Seção 1.

CAPORAL, F. R. et al. As bases epistemológicas da agroecologia. Paraná: INSTITUTO FEDERAL DE EDUCAÇÃO, CIÊNCIA E TECNOLOGIA DO PARANÁ, 2011.

CORDEIRO, K. et al. A Tecnologia Social e o Modo de Produção de Hortaliças da Comunidade Quilombola Chácara Buriti, em Campo Grande - MS. Revista Sociedade Brasileira de Economia, Administração e Sociologia Rural (SOBER). Campo Grande, 2010.

EHLERS, E. Agricultura Sustentável: origens e perspectivas de um novo paradigma. Guaíba: Editora Agropecuária.2. Ed., 1999. 
FIGUEIREDO, E. A. P. De; SOARES, J. P. G. Sistemas orgânicos de produção animal: dimensões técnicas e econômicas. In: Reunião Anual da Sociedade Brasileira de Zootecnia, 49, Brasília 2012.

FUNDAÇÃO BANCO DE BRASIL. Tecnologia Social na Fundação Banco do Brasil: Soluções para o Desenvolvimento Sustentável. Brasilia, 2009.

IRIAS, L. J. et al. Sistema de avaliação de impacto ambiental de inovações tecnológicas nos segmentos agropecuário, produção animal e agroindústria (sistema AMBITEC). Circular técnica Embrapa. Brasil. 2004.

KHATOUNIAN, C. A. A reconstrução ecológica da agricultura. Botucatu: Agroecológica, 2001.

MEIRELLES, M. R.; Rupp, L.C. Agricultura ecológica. Princípios básicos. Ministério de desenvolvimento agrário Centro ecológico. Secretaria da agricultura familiar. Brasil, 2005.

NEVES, D. A. L. Escolhas estratégicas para produção de carne bovina orgânica no Brasil. Dissertação (Mestrado em Agronegócios). UnB, 2012.

PAVAN, M. et al. Tecnologia Social, agroecologia e agricultura familiar: análises sobre um processo sociotécnico. Revista Segurança Alimentar e Nutricional. São Paulo. 8, 169-181, 2013.

RODRIGUES, G. S. et al. Avaliação de Impacto Ambiental da Inovação Tecnológica Agropecuária: AMBITEC-AGRO. Jaguariúna: Embrapa Meio Ambiente, 2003. 95p

. Avaliação de impactos ambientais na agropecuária. Gestão Ambiental na Agropecuária. Embrapa Informação Tecnológica. Brasília, 2006.

ROMÃO, M. A. Produção Agroecológica Integrada e Sustentável (PAIS), uma tecnologia social para construção da segurança alimentar. In: Morais, L.; Borges, A. Novos paradigmas de produção e consumo Experiências inovadoras. São Paulo. Instituto Pólis, 2010. f. $56-98$.

SOUZA, A. A. et al. Agroecologia. In: L (Org.); Agroecologia. Centro Col ed., 2012.

SERVIÇO BRASILEIRO DE APOIO ÀS MICRO E PEQUENAS EMPRESAS - SEBRAE. Produção Agroecológica Integrada e Sustentável - PAIS. Brasil, 2006. $20 \mathrm{f}$.

SOARES, J.P.G. et al. Impactos Ambientais da Transição entre a Produção de Leite Bovino Convencional para Orgânico na Região Integrada de Desenvolvimento do Distrito Federal e Entorno (RIDE/DF). Boletim de Pesquisa e Desenvolvimento 324. Brasília DF:Embrapa, 2015.

SOARES, J.P.G; Rodrigues, G.S. Avaliação social e ambiental de tecnologias Embrapa: Metodologia Ambitec-Agro. In: Workshop em Avaliação Econômica de Projetos e Impactos de Tecnologias da Embrapa. Pereira, M.A; Malafaia, G.(Org) Campo Grande: Embrapa Gado de Corte, 2013. 95p. (Documentos 205) (ISSN 1983-974X)

VENTURA, A; GARCIA, L.; ANDRADE, J. Tecnologias sociais: as organizações não governamentais no enfrentamento das mudanças climáticas e na promoção do desenvolvimento humano. Cadernos EBAPE.BR, v.10,n.3,2012. 